\title{
Priming from novel masked stimuli depends on target set size
}

\author{
Andrea Kiesel ${ }^{1}$, Wilfried Kunde ${ }^{2}$, Carsten Pohl ${ }^{1}$, and Joachim Hoffmann ${ }^{1}$ \\ ${ }^{1}$ Department of Psychology, Julius-Maximilians University of Würzburg, Germany \\ 2 Department of Psychology, Martin-Luther-University Halle-Wittenberg, Germany
}

Received 29.03.2005

Accepted 07.07.2005

\section{Keywords}

Masked priming, novel stimuli, action trigger account

\begin{abstract}
When objects denoted by target words are classified as belonging to a certain category (e.g., to be either small or large) responding is faster when the target word is preceded by a masked prime word belonging to the same rather than a different category. Recently, there has been some controversy on whether such masked priming effects are confined to primes that are practised as targets as well, or whether they transfer to other

novel prime words. We report data which show that the transfer of unconscious priming to unpractised stimuli depends on the size of the target set. Priming does transfer to novel (unpractised) primes with a large target set (40 different target words), whereas no transfer to novel primes occurs with a small target set (4 different target words). We conclude that the size and structure of the target set crucially determine the way participants handle a task and thus, determine how unconscious stimuli are processed.
\end{abstract}

Masked response priming is a well-established method for investigating the processing of unconscious stimuli. Typically, participants perform a speeded two-choice response task, for example, they indicate whether a presented word denotes an object that is small or large (Damian, 2001). Prior to the target word another stimulus, the so-called prime, is presented for a short duration so that participants remain unaware of it. The prime is either congruent, that is, it affords the same response, or incongruent, that is, it affords the opposite response, to the target. Faster response times (and lower error rates) in trials with congruent compared to trials with incongruent primes indicate unconscious prime processing.

Recently, the mechanisms underlying masked response priming have attracted considerable interest (e.g., Ansorge, Heumann, \& Scharlau, 2002; Dehaene, et al., 1998; Dell'Acqua \& Grainger, 1999; Klinger, Burton, \& Pitts, 2000; Kunde, Kiesel, \& Hoffmann, 2003; Mattler, 2003; Neumann \& Klotz, 1994; Snodgrass, Bernat, \& Shevrin, 2004). A powerful instrument for learning about these mechanisms is the assessment of priming from novel unseen stimuli. Here, the crucial question is whether priming effects are confined to stimuli that are also practiced (and are responded to) as targets or whether they transfer to novel prime words as well. Priming effects from novel prime stimuli would indicate that priming goes beyond activating acquired S-R (stimulus-response) associations. In fact, priming from novel stimuli often has been taken as evidence for semantic processing of unconscious stimuli (e.g., Dell'Aqua \& Grainger, 1999; Greenwald, Abrams, Naccache, \& Dehaene, 2003; Naccache \& Dehaene, 2001; Reynvoet, Caessens, \& Brysbaert, 2002; Van Opstal, Reynvoet, \& Verguts, in press), though alternative interpretations might hold as well (cf. Kunde, Kiesel, \& Hoffmann, 2003).

Correspondence concerning this article should be addressed to Andrea Kiesel, Julius-Maximilians University of Würzburg, Institut für Psychologie III, 97070 Würzburg, Germany. Electronic mail may be sent via Internet to kiesel@psychologie. uni-wuerzburg.de 


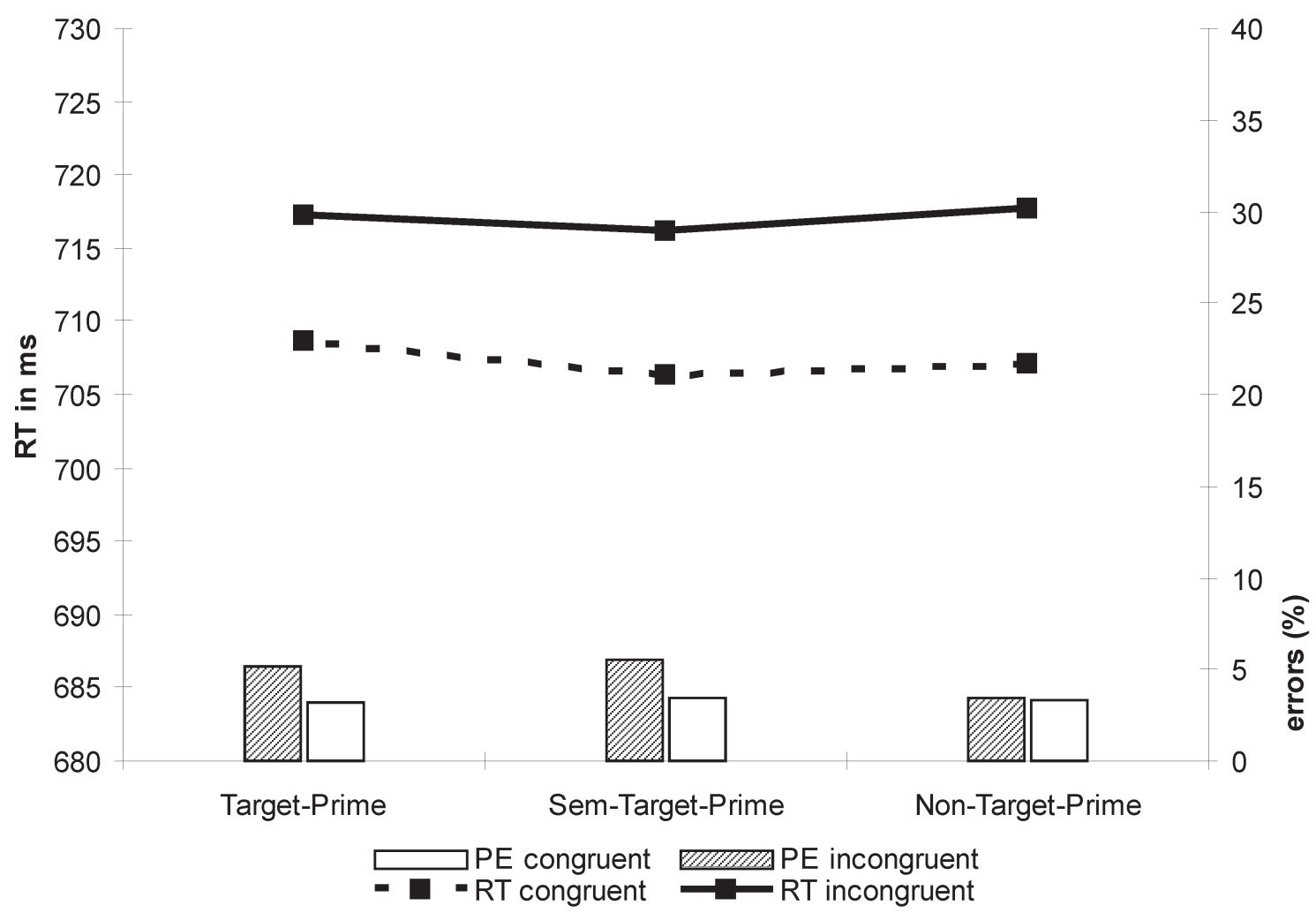

Figure 1

Large target set (Exp 1). Mean RTs and error rates for incongruent (filled) and congruent (dotted) primes depending on Prime Type.

For numeral stimuli such novel-prime effects have repeatedly been observed (e.g., Greenwald, et al., 2003; Kunde, et al., 2003; Naccache \& Dehaene, 2001; Reynvoet, et al., 2002; Van Opstal, et al., in press). Remarkably, for word stimuli the evidence is more limited in number and, up to now, clearly negative (Abrams \& Greenwald, 2000; Damian, 2001). For example, in the study by Damian (2001) participants classified words according to the size of the objects denoted by them to be either smaller or larger than a $20 \times 20 \times 20 \mathrm{~cm}$ reference frame. Prime words affected performance only when they were used as targets as well, and they did so more strongly the more frequently they were responded to. The straightforward conclusion from these results would be that masked numeral stimuli (including numerals) are processed semantically, whereas masked word stimuli are not.

From the available evidence it is hard to judge what the reasons for the lack of priming from novel words might be. Here we explore the possibility that processing of novel prime stimuli is determined by the number of target stimuli. Specifically, priming might remain confined to practiced stimuli with small sets of target stimuli, as this allows responding according to specific S-R instances, whereas priming does extend to novel stimuli with large target sets that render an instance-based way of responding less favourable. Two explanations of this supposed small-set large-set difference are considered in the general discussion: One that assumes unconscious semantic processing that is blocked in the special case of small target sets and another that accounts for the results without assuming unconscious semantic processing.

We report two experiments. In Experiment 1, with a large target set, priming did extend to novel primes, whereas in Experiment 2 using the same task but a limited target set, it did not.

\section{EXPERIMENT 1}

Noun words referring to concrete objects served as stimuli. Participants were asked to categorize the target as being smaller or larger than a reference object by pressing a left or a right response key. In each trial a masked prime word was presented prior to the target word. This prime word required either the same (congruent) or the other (incongruent) response as the target. Prime processing was assessed by performance differences for congruent and incongruent primes. Three different types of primes were used: Primes that were also used as targets (Target-Prime), primes that were not used as targets but that were semanti- 


\section{Table 1}

Targets used in Experiment 1 (and their English translations)

\begin{tabular}{llll}
\hline Small category & & Large category & \\
\hline KNOPF (button) & LÖFFEL (spoon) & HAUS (house) & BERG (mountain) \\
MESSER (knife) & WÜRFEL (dice) & TISCH (table) & KIRCHE (church) \\
BECHER (mug) & NAGEL (pin) & AUTO (car) & TRAKTOR (tractor) \\
PINSEL (brush) & RING (ring) & BET (bed) & HOTEL (hotel) \\
ZIGARRE (cigar) & KAMM (comb) & SCHIFF (ship) & DOM (cathedral) \\
PFEIFE (pipe) & BRIEF (letter) & TURM (tower) & SESSEL (armchair) \\
BATTERIE (battery) & SCHERE (pair of scissors) & TÜR (door) & ZELT (tent) \\
KERZE (candle) & SCHRAUBE (screw) & SCHRANK (wardrobe) & SOFA (sofa) \\
APFEL (apple) & MÜNZE (coin) & TEPPICH (carpet) & BUS (bus) \\
TASSE (cup) & NADEL (needle) & KOFFER (suitcase) & PALAST (palace) \\
\hline
\end{tabular}

\section{Table 2}

Primes used in Experiment 1 and 2 (and their English translations)

\begin{tabular}{lll}
\hline Target primes & Sem-Target primes & Non-Target primes \\
\hline KNOPF (button) & GABEL (fork) & BRILLE (glasses) \\
HAUS (house) & STUHL (chair) & ZUG (train) \\
\hline
\end{tabular}

cally related to some targets (Sem-Target-Prime), and primes that were not used as targets and that were semantically unrelated to the target (Non-Target-Prime). We were interested in whether priming effects transfer to novel prime stimuli, thus, to the Prime Types Sem-Target and Non-Target. The manipulation of semantic relatedness was incorporated to assess the extent of semantic processing of the primes. A semantic analysis should show up as faster responding with primes that are not only congruent but also semantically related (rather than unrelated) to the targets, similar to what has been reported for numerical distance with number stimuli (e.g., Koechlin, Naccache, Block, \& Dehaene, 1999).

\section{Method}

\section{Participants}

Twelve volunteers (aged 19-42) took part in individual sessions of approximately $40 \mathrm{~min}$ either in fulfilment of course requirements or in exchange for pay. All participants reported having normal or corrected-to-normal vision, and were not familiar with the purpose of the experiment.

\section{Apparatus and Stimuli}

An IBM compatible computer with a 17 inch VGA-display and the software package E-Prime (Schneider, Eschman, \& Zuccolotto, 2002) was used for stimulus presentation and response sampling. Stimulus presentation was synchronized with the vertical retraces of the
$70-\mathrm{Hz}$ monitor, resulting in a refresh rate of $14.3 \mathrm{~ms}$. Responses were executed with the index fingers of both hands and collected with an external keyboard.

The target set consisted of forty words (see Table 1) naming objects that are clearly smaller or larger than the reference object (a soccer ball). Six words (see Table 2) and a neutral letter string (either NPXQH or NPXLH) were used as primes. The six prime words either came from the target word set (Target-Primes), were closely semantically related to two target words (Sem-Target-Primes, "chair" and "fork" are closely related to the targets "table" and "knife") ${ }^{1}$ or did not belong to the target set (Non-Target-Primes). Thus, the stimulus set consisted of 280 prime-target-pairs that were presented once per block. Participants performed in two blocks. Between the blocks participants were allowed a short, self-paced break.

In each trial, the prime was presented for $43 \mathrm{~ms}$ (3 refresh cycles). It was preceded and followed by random letter masks presented for $72 \mathrm{~ms}$. The target was presented directly after the post-mask for $200 \mathrm{~ms}$. All stimuli were presented centered on the screen in white on a black background in Courier New 36.

In a post-experimental detection task another 120 trials were presented. Half of these signal detection trials contained the neutral non-word prime.

\section{Design and Procedure}

Participants were instructed to categorize the presented target words as smaller or larger than a soccer ball. They were not informed about the presence 


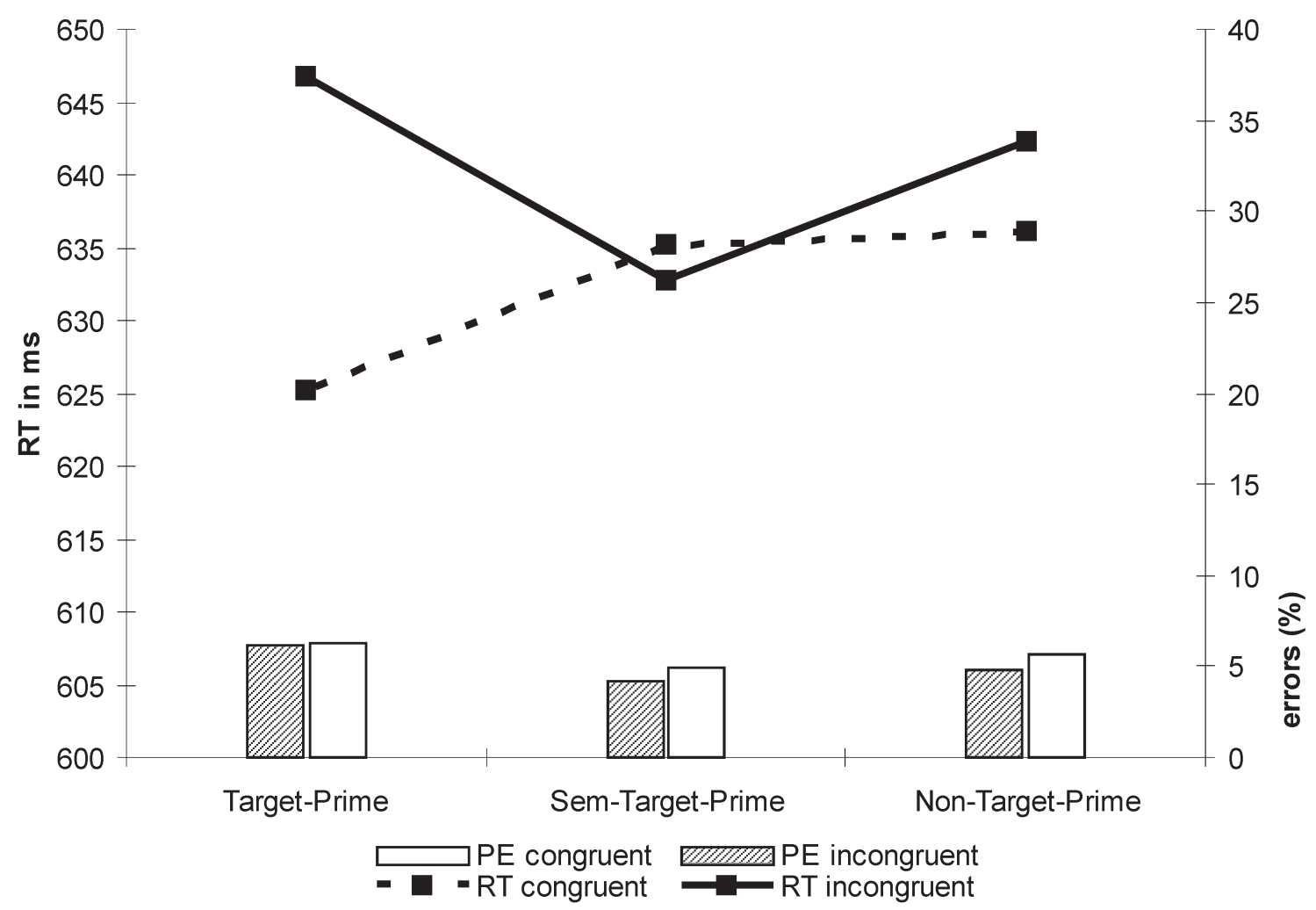

Figure 2

Small target set (Exp. 2). Mean RTs and error rates for incongruent (filled) and congruent (dotted) primes depending on Prime Type.

of primes. Half of the participants pressed a left key with the left index finger to indicate "smaller" and a right key with the right index finger to indicate "larger" as fast and as accurately as possible. For the other half of the participants the mapping was reversed. Errors were indicated by the German word for wrong ("Falsch!") presented in red in the lower part of the monitor. Response times were recorded from the onset of the target until the onset of the response.

After the experiment, participants were fully informed about the precise structure of the prime stimuli. They were then presented with 120 trials identical to the experimental trials. 60 trials contained the neutral prime. Participants were to discriminate whether a prime or the neutral random letter string was presented by pressing the 1 or the 0 of the number keyboard. For the discrimination task, participants were instructed to take their time and to try to be as accurate as possible.

\section{Results}

\section{Prime visibility}

Data from one participant were discarded from further analysis due to an exceptional high $d^{\prime}$ value of 1.53 (the second highest value was $d^{\prime}=0.68$ ). For the remaining participants the discrimination performance for neutral vs. non-neutral primes was $d^{\prime}=0.17$ (the mean hit rate was $61.7 \%$, mean false alarm rate was $55.0 \%$ ) and deviated from zero, $t(10)=1.87, p<.05$ (one-tailed).

\section{Congruency effect}

Trials with RTs (reaction times) deviating more than 2.5 standard deviations from the mean RT of each participant and each condition were excluded. Mean RTs for correct responses were submitted to an ANOVA with the within-subject factors Prime Type (Target-Prime, Sem-Target-Prime, and Non-Target-Prime) and Congruency (congruent and incongruent ${ }^{2}$ ). The factor Congruency was significant, $F(1,10)=8.2, p<.05$, $M S E=1,534.3$. Participants responded faster for congruent (707 ms) compared to incongruent (717 ms) primes (see Figure 1). The factor Prime Type was not significant and did not interact with Congruency ( $p$ s > .94).

The same ANOVA on error rates revealed a marginally significant effect for Congruency, $F(1,10)=4.7$, $p=.055$, MSE $=.00342$, indicating that participants responded erroneous more frequently in incongruent $(4.7 \%)$ compared to congruent (3.3\%) trials. Neither the factor Prime Type nor the interaction of Prime Type $x$ Congruency approached significance ( $p s>.31$ ). 


\section{Discussion}

Participants responded slower and were more error prone with incongruent compared to congruent primes regardless of whether the primes were practiced as targets or not. To the best of our knowledge this is the first report of response priming from novel primes with word stimuli.

But why then did we observe priming for novel word-stimuli, whereas other studies did not (Abrams \& Greenwald, 2000; Damian, 2001)? First of all it seems worthwhile to consider, that our masking procedure was not sufficiently efficient. Mean $d^{\prime}$ to detect the primes was 0.17, whereas in a comparable Experiment of Damian (2001, Exp. 2) it was 0.078. For some reason a spread of priming to unseen stimuli might depend on efficiency of masking. However, a lack of transfer was also observed in the study by Abrams and Greenwald (2000, Exp. 3) where the individual $d^{\prime}$ values ranged from -0.3 to 1.6 and even participants with high $d^{\prime}$ values showed no priming effect. Moreover, the individual $d^{\prime}$ values and the size of the priming effect we observed did not correlate with each other $(r=-.046, p>.89)$. Even participants with low $d^{\prime}$ values elicited masked priming effects. Therefore, we found the assumption not very convincing that a slight variation in visibility determines whether priming transfers to novel stimuli.

A second and, in our view, more notable difference between our study and the other reports concerns the size of the target set. In our study, 40 different targets were presented, whereas the target sets in the studies by Abrams and Greenwald (2000, Exp. 3) and Damian (2001) were notably smaller, namely of the size 16 respectively 12 . As noted in the introduction, the number of presented targets might be crucial for a transfer of priming to novel stimuli. Conceivably, the lower the number of distinguishable targets, the easier it is to recollect a recent S-R episode. In other words, with a small target set it might suffice to discriminate the presented target from the remaining potential targets and to remember its assigned response (Logan, 1988). This discrimination does not necessarily require a semantic evaluation but can be achieved by peripheral (e.g., perceptual) properties as well. From this perspective, Non-Target primes fail to evoke priming effects, because they do not match the few experienced targets at a perceptual level. Such a scenario is certainly conceivable for a target set of, say, two words, but it might well extend to sets of 12 or 16 targets as have been used by Damian (2001) and Abrams and Greenwald (2000). To corroborate this inference we conducted another experi- ment, in which under otherwise the same conditions the number of targets was substantially reduced. We expected priming to be confined to primes from the target set under these conditions.

\section{EXPERIMENT 2}

\section{Method}

\section{Participants}

Twelve volunteers (aged 19-32) each took part in an individual session of approximately 40 min either in fulfilment of course requirements or in exchange for pay. All participants reported having normal or corrected-to-normal vision, and were not familiar with the purpose of the experiment.

\section{Apparatus and Stimuli}

Apparatus and stimuli were identical to Experiment 1 expect that only four target words were used as stimuli (the words MESSER [knife], KNOPF [button], TISCH [table], and HAUS [house]). Thus, there were 28 possible prime-target-combinations which were presented 10 times per block. Again participants performed two blocks separated by a short break.

\section{Design and Procedure}

Design and procedure were identical to Experiment 1.

\section{Results}

\section{Prime visibility}

Discrimination for neutral vs. non-neutral primes was $d^{\prime}=0.24$ (the mean hit rate was $51.1 \%$, false alarm rate $41.8 \%$ ) and deviated from zero, $t(11)=3.5$, $p<.01$. More importantly for the purpose of the study, the $d^{\prime}$ values of Experiment 1 and Experiment 2 did not differ from each other, $t(21)=0.55, p=.59$ (two-tailed).

\section{Congruency effects}

Trials with RTs deviating more than 2.5 standard deviations from the mean RT of each participant and each condition were excluded. Mean RTs for correct responses were submitted to an ANOVA with the within-subject factors Prime Type (Target-Prime, Sem-Target-Prime, and Non-Target-Prime) and Congruency (congruent and incongruent $^{3}$ ). The factor Congruency was significant, $F(1,11)=12.5, p<.01$, MSE $=1,278.0$, as well as the interaction Prime Type $x$ Congruency, $F(2,22)=3.6$, $p<.05, M S E=879.7$. The main effect for Prime Type did not approach significance $(p>.32)$.

Figure 2 shows that there was a congruency effect for Target-Primes: Participants responded faster for congruent (625 ms) compared to incongruent Target-Primes, 

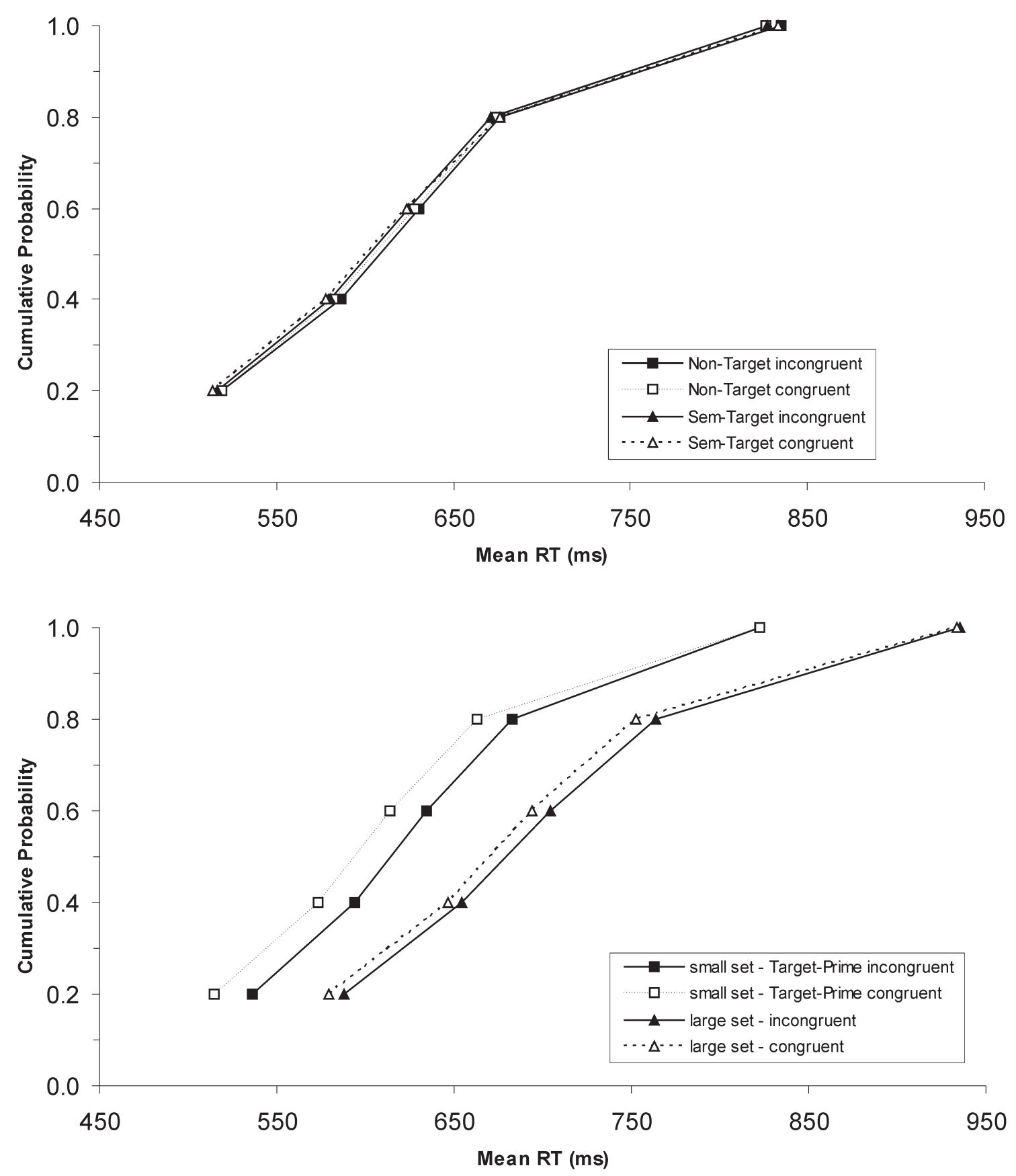

\section{Figure 3}

Cumulative density functions. Top panel shows congruent and incongruent trials for Non-Target-Primes and Sem-Target-Primes (Exp. 2), bottom panel shows congruent and incongruent trials for Target-Primes of the small target set (Exp. 2) and congruent and incongruent trials for the large target set (Exp. 1, averaged over all Prime Types).

$647 \mathrm{~ms}, t(11)=3.5, p<.01$. There was no congruency effect for the other two prime types ( $p s>.35$ ).

The same ANOVA for error rates revealed no significant effect at all ( $p s>.23)$.

\section{Discussion}

In Experiment 2 priming was confined to primes that were practised as targets. Thus, Experiment 2 rep- licates previous results of Abrams and Greenwald (2000) and Damian (2001). For novel prime stimuli no priming was observed even when the primes were closely semantically related to the targets. Hence there was no evidence for any access to semantic codes in Experiment 2. We argue that responding to a limited target set can be based on remembering specific S-R instances, so that a semantic analysis of stimuli becomes superfluous. 
Alternatively, one may argue that the lack of priming in Experiment 2 results from the generally lower RT level. To explore this possibility, we conducted a distribution analysis for the RTs from Experiment 1 and 2 (see Figure 3). For Experiment 2, congruent and incongruent trials were analysed separately for each Prime Type, for Experiment 1, all Prime Types were analysed together as this factor did not affect the priming effect. If semantic priming needs time to unfold, one would expect a priming effect for Non-Target-Primes and Sem-Target-Primes to emerge for long RTs in Experiment 2. Furthermore, the observed priming effect for Target-Primes should increase with RTs as the semantic priming effect adds to the priming via S-R associations. And finally, the priming effect observed in Experiment 1 should be increased for long RTs.

Figure 3 shows that neither of these expectations is confirmed. No priming effect arises for the Non-Target-Primes and the Sem-Target-Primes for long RTs in Experiment 2 (see Figure 3, top panel). And the observed priming effects (for Target-Primes in Exp. 2 and the general effect in Exp. 1) do not increase for long RTs (see Figure 3 bottom panel). Thus, we can rule out that semantic priming was not measurable in Experiment 2 because participants responded too fast. Interestingly, the size of the priming effect for the Target-Primes was substantially increased in Experiment 2 compared to Experiment 1 ( $22.5 \mathrm{~ms}$ vs. $8.5 \mathrm{~ms}$ ). This additionally supports the assumption that in Experiment 2 priming is mediated by well-trained, acquired S-R associations (cf. Greenwald, Draine, \& Abrams, 1996).

\section{GENERAL DISCUSSION}

Masked priming has been shown to transfer to novel word-stimuli for a large target set (Experiment 1), whereas it was confined to practised target-words with a small target set (Experiment 2). A somewhat similar result concerning the impact of "novel" word stimuli as in Experiment 1 was recently reported by Naccache, et al. (2005). In this study, epileptic patients were to indicate whether a word is threatening or nonthreatening with the words either supra- or subliminally presented. The neuronal activity of the amygdala was recorded in response to word presentation by intracranial electrodes. The data revealed that the amygdala responded also to words which were exclusively subliminally presented suggesting that the threatening potential of these "novel" words were recognized despite being never consciously experienced as targets. Interestingly, like in our Experiment 1, the target set comprised a large number of words (92 in each category). Thus, semantic aspects of subliminally presented words seem to be available if participants respond to a large set but not if they respond to a small set of supraliminal target words.

Taken together, our results can be accounted for in two different ways. First, one may assume that the findings seem to point to two different processing modes for subliminally presented word-stimuli: In case of a small target set, words may prime responses by a reactivation of recently experienced concrete S-R episodes. In this mode the subliminal words do not need to be semantically analyzed. Instead, it suffices to detect that a presented stimulus matches a recently passed one so that priming remains restricted to target-primes. In case of a large target set, however, such a response mode would be useless. Consequently, participants may adopt another mode, namely stimulus words receive a more elaborate processing including an analysis of the semantic features so that also novel prime words affect responses.

However, there are some aspects of our data which are not entirely consistent with this account. Most notably, in Experiment 2, the priming effects from the target-primes should increase the stronger the S-R associations are, that is, the more often participants responded to a target with the corresponding response. The data, however, provided no hint for an increase of priming as priming effects amounted to $23 \mathrm{~ms}, 26 \mathrm{~ms}, 10 \mathrm{~ms}$, and $27 \mathrm{~ms}$ respectively in the first, second, third and fourth quarter of trials. Of course such an analysis does not allow to rule out that stimuli prime responses by reactivating recently experienced S-R episode as one single trial may suffice to build up a S-R association.

On the other hand a semantic analysis of the novel primes in Experiment 1 is also questionable as the variation of semantic relatedness of non-target primes to targets had no impact on priming. For example, the prime "Gabel (fork)" did not facilitate responding to the target "Messer (knife)" as compared to the prime "Knopf (button)" despite the former being more closely related to the target than the latter ${ }^{4}$. Thus, whatever analysis was carried out on the primes, it did certainly not encompass all the meanings the primes convey.

Finally, the assumption of two different processing modes contravenes the criterion of parsimony. All these considerations let us prefer an alternative account which assumes a common processing mode for large as well as small target sets:

According to our view, participants categorize (off-line) the to-be-expected target-stimuli into appropriate 
and inappropriate release conditions for the required responses. For example, when asked to categorize objects as being smaller or larger than a soccer ball, memory codes of appropriate objects or object categories are preactivated and assigned to the respective response. Such a preactivation of memory codes of expected objects might well be possible for very broad semantic categories, such as for words with a specific gender (cf., Bates, Devescovi, Pizzamiglio, D’Amico, \& Hernandez, 1995). The preparatory activations in turn reduce the amount of perceptual evidence needed to classify the prime according to the required response selection criteria. In order to give an illustrative example: When asked to decide whether an upcoming word denotes the name of famous poet or a famous pop-singer the word fragment Sh_k_spe_r_ might be rapidly classified as poet before (or even without) being fully identified.

Based on work by Ach (1905), Neumann (1989) and others we recently introduced the term "action triggers" for such preactivated memory codes (cf. Kunde, et al., 2003). Concerning subliminal priming settings, we assume that subliminal stimuli activate responses only according to existing action triggers, that is, by a comparison between the preactivated memory codes and the current perceptual input.

If many different targets are to be expected, the preparatory activation of memory codes can encompass broad categories. However, if less broad categories work as well the "action triggers" are likely narrowed down. This probably happens when the target set is small, that is, when preactivation can be easily restricted to few particular targets.

This account has the favourable aspect that it does not assume different modes of prime processing. Given that a memory code is activated, a match of a stimulus with that code can be established even by virtue of subliminal perceptual evidence without semantic processing. This lack of semantic prime analysis would also explain the lack of any effect of semantic primetarget relatedness in Experiment 1. Although these speculations require further proof they appear to us a reasonable working hypothesis that can account for a couple of otherwise contradictory results in masked priming (Kunde, Kiesel, \& Hoffmann, 2003; in press).

Nevertheless, there remain issues to be clarified. To mention one of them: Why did we not find transfer of priming to novel, unseen stimuli with a set of four word targets, whereas such transfer occurs with sets of four number targets? For example, when asked to classify the numbers $1,4,6$, and 9 as smaller or larger than 5, the primes $2,3,7$, and 8 exert priming effects as well, though they were never presented as targets them- selves (Naccache \& Dehaene, 2001). The contradiction can be likely ascribed to differences in the representation of numbers and words. Numbers are represented in a tightly associated manner like a mental number line (e.g., Galton, 1880; Göbel, Walsh, \& Rushworth, 2001). The activation of certain numbers typically spreads over to adjacent elements. Consequently, the preactivation of only some certain numbers as "triggers" (e.g., the numbers 1 and 4 ) will make it very hard to prevent numerically enclosed numerals (2 and 3 ) from entering the same trigger set. In contrast, semantic memory is much more heterogeneous and multidimensional than the mental number line. Therefore, the spread of activation to semantically related codes is less likely in case of words than in case of numbers.

In conclusion, we have shown that the size of the target set is a factor that affects the transfer of priming to novel unseen primes. We suggest that the size of target set determines which features are used to specify response release conditions. With large target sets, these features are broad, semantically defined, and result in preactivation of many potential stimulus instances. With small target sets, these features are fine-tuned, perceptual, and result in preactivation of only a few stimulus instances. Alternative explanations in terms of different processing pathways depending on the size of the target set seem possible as well, but appear not to be strongly supported by the available data reported here. Future research will have to clarify which of these concepts portrays the mechanisms of unconscious priming most adequately.

\section{Acknowledgements}

This research was funded through Deutsche Forschungsgemeinschaft Grant HO 1301/10-1 awarded to Joachim Hoffmann and Grant KU 1964/1-1 to Wilfried Kunde.

\section{Notes}

${ }^{1}$ The distinction between Non-Target-Primes and Sem-Target-Primes may not appear very convincing when using forty targets. However, in Experiment 2 this distinction becomes important. For better comparability of both experiments we make this distinction here, too. But the results of the analysis are similar when Non-Target-Primes and Sem-Target-Primes were analysed together.

2 The data from trials with neutral primes cannot be included in the analysis as no variation of Prime Type is possible for neutral primes. The mean RTs and error rates for neutral primes were $720 \mathrm{~ms}$ and $5.5 \%$.

3 The mean RTs and error rates for neutral primes were $639 \mathrm{~ms}$ and $4.4 \%$. 
${ }^{4} \mathrm{~A}$ reanalysis of Experiment 1 showed that response times and error rates for semantically related primes do not differ irrespective of whether the prime word "Gabel" is presented prior to the target word "Messer" and "Stuhl" prior to "Tisch" compared to when the prime word "Gabel" is presented prior to "Knopf" and "Stuhl" prior to "Haus" (RTs: $t(10)=0.26$, PEs: $t(10)=-0.43)$. However, one has to be cautious with this analysis as for this specific analysis there are only four trials per subject and condition.

\section{REFERENCES}

Abrams, R. L., \& Greenwald, A. G. (2000). Parts outweigh the whole (word) in unconscious analysis of meaning. Psychological Science, 11, 118-124. Www

Ach, N. (1905). Über die Willenstätigkeit und das Denken. Göttingen: Vandenhoeck \& Ruprecht.

Ansorge, U., Heumann, M., \& Scharlau, I. (2002). Influences of visibility, intentions, and probability in a peripheral cuing task. Consciousness and Cognition, 11, 528-545. $\mid \mathrm{wWw}$

Bates, E., Devescovi, A., Pizzamiglio, L., D'Amico, S., \& Hernandez, A. (1995). Gender and lexical access in Italian. Perception \& Psychophysics, 57, 847-862. Www

Damian, M. F. (2001). Congruity effects evoked by subliminally presented primes: Automaticity rather than semantic processing. Journal of Experimental Psychology: Human Perception and Performance, 27, 154-165. |www|

Dehaene, S., Naccache, L., Le Clec`H, G., Koechlin, E., Mueller, M., Dehaene-Lambertz, G., van de Moortele, P.-F., \& Le Bihan, D. (1998). Imaging unconscious semantic priming. Nature, 395, 597-600. www

Dell' Acqua, R., \& Grainger, J. (1999). Unconscious semantic priming from pictures. Cognition, 73, B1-B15. |WWW

Galton, F. (1880). Visualised numerals. Nature, 21, 252-256.

Göbel, S., Walsh, V., \& Rushworth, M. F. S. (2001). The mental number line and the human angular gyrus. NeuroImage, 14, 1278-1289. [WWW

Greenwald, A. G., Abrams, R. L., Naccache, L., \& Dehaene, S. (2003). Long-term semantic memory versus contextual memory in unconscious number processing. Journal of Experimental Psychology: Learning, Memory, and Cognition, 29, 235-247. WWw

Greenwald, A. G., Draine, S.C., \& Abrams, R. L. (1996). Three cognitive markers of unconscious semantic activation. Science, 273, 1699-1702. |WWW

Klinger, M. R., Burton, P. C., \& Pitts, G. S. (2000). Mechanisms of unconscious priming: I. response competition, not spreading activation. Journal of Experimental Psychology: Learning, Memory, and Cognition, 26, 441-455. (www)

Koechlin, E., Naccache, L., Block, E., \& Dehaene, S. (1999). Primed numbers: Exploring the modularity of numerical representations with masked and unmasked semantic priming. Journal of Experimental Psychology: Human Perception and Performance, 25, 1882-1905.

Kunde, W., Kiesel, A., \& Hoffmann, J. (2003). Conscious control over the content of unconscious cognition. Cognition, 88, 223-242. $\underline{\mathrm{WW}}$

Kunde, W., Kiesel, A., \& Hoffmann, J. (in press). On the masking and disclosure of unconscious elaborate processing. A reply to Van Opstal, Reynvoet, \& Verguts (2005). Cognition. $\underline{\underline{w} w \mid}$

Logan, G. D. (1988). Toward an instance theory of automatization. Psychological Review, 95, 492-527.

Mattler, U. (2003). Priming of mental operations by masked stimuli. Perception \& Psychophysics, 65, 167-187. WWw

Naccache, L., \& Dehaene, S. (2001). Unconscious semantic priming extends to novel unseen stimuli. Cognition, 80, 223-237. $\mid \mathrm{ww}$

Naccache, L., Gaillard, R., Adam, C., Hasboun, D., Clemenceau, S., Baulac, M., Dehaene, S., \& Cohen, L. (2005). A direct intracranial record of emotions evoked by subliminal words. PNAS, 102, 7713-7717. |WwW|

Neumann, O. (1989). On the origins and status of the concept of automatic processing. Zeitschrift für Psychologie, 197, 411-428.

Neumann, O., \& Klotz, W. (1994). Motor responses to nonreportable, masked stimuli: Where is the limit of direct parameter specification? In C. Umilta \& M. Moscovitch (Eds.), Attention and performance XV. Conscious and nonconscious information processing (pp. 123-150). Cambridge: MIT Press.

Reynvoet, B., Caessens, B., \& Brysbaert, M. (2002). Automatic stimulus-response associations may be semantically mediated. Psychonomic Bulletin \&

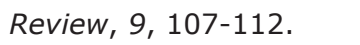

Schneider, W., Eschman, A., \& Zuccolotto, A. (2002). E-prime user's guide. Pittsburgh: Psychology Software Tools Inc.

Snodgrass, M., Bernat, E., \& Shevrin, H. (2004). Unconscious perception: A model based approach to method and evidence. Perception \& Psychophysics, 66, 846-867. $\overline{w w w \mid}$

Van Opstal, F., Reynvoet, B., \& Verguts, T. (in press). How to trigger elaborate processing? A comment on Kunde, Kiesel, and Hoffmann (2003). Cognition. Www 